\title{
Physicians’ Perception of Drug Information Resources in the Emirate of Abu Dhabi-UAE ${ }^{*}$
}

\author{
Hadaya Gharibyar $^{1 \#}$, Yasser Sharif ${ }^{2}$, Kefah Al Qawasme ${ }^{1}$, Sahar Fahmy ${ }^{3}$ \\ ${ }^{1}$ Medication Safety Officer, Health Authority, Abu Dhabi, UAE; ${ }^{2}$ Head Medication \& Medical Products Safety Section, Health Au- \\ thority, Abu Dhabi, UAE; ${ }^{3}$ Assistant Professor, College of Pharmacy, Helwan University, Cairo, Egypt. \\ Email: "hgharibyar@haad.ae
}

Received October $24^{\text {th }}, 2012$; revised November $30^{\text {th }}, 2012$; accepted December $16^{\text {th }}, 2012$

\begin{abstract}
Objectives: To determine physicians’ preferences for different drug information resources in the United Arab Emirates. Methods: A questionnaire survey to determine preferences for different drug information resources was created and sent electronically from April 1 to May 13, 2012. A total of 3463 licensed physicians were identified from a comprehensive list of healthcare professionals provided by the Health Authority-Abu Dhabi Licensing Department. A total of 431 started the survey but only 372 physicians completed it entirely. Key Findings: Continuing medical education (46\%) was ranked as the most favored source of information, followed by drug information references (28\%), diagnosis/patient information (20\%), and lastly peer-to-peer interactions (7\%). Online sources of drug information were preferable (74\%) to hardcopy (26\%). A comparison of different physicians' qualifications (consultant, specialist, and general practitioner) and number of references utilized for a particular topic (up to 3 resources versus 3 references or more) showed that their differences were statistically significant ( $p$-value $=0.000$ ). The most utilized sources of drug information by physicians was the British National Formulary (BNF), followed by Package Inserts, UpToDate, Drugs in Pregnancy \& Lactation, Physicians' Desk Reference, and Micromedex. When asked which drug information resource they would like to have access to, the majority of physicians choose the BNF, followed by UpToDate, MD Consult, Drugs in Pregnancy \& Lactation, Micromedex, and Drugs Facts \& Comparisons. Physicians from Europe/USA/Australia spent less time utilizing drug information resources (p-value of 0.013) compared to other nationalities. Conclusion: Preferences for different drug information resources did not vary significantly even amongst a diverse group of expatriate physicians licensed to practice in the United Arab Emirates.
\end{abstract}

Keywords: Drug Information; Abu Dhabi; United Arab Emirates; Physicians; Medical Information

\section{Introduction}

Physicians across the world use different sources of drug information such as newsletters, pharmaceutical drug information brochures, pharmacists, continuing medical education, text books, conferences/seminars, and literature. Sometimes the preferential choice could be guided by whether the source is available online or through a portable electronic device or hardcover. The dissemination of new medical information and incorporation of research findings into practice by health professionals are major challenges in the field of medicine [1,2].

Demographic and practice characteristics are debated as factors in physicians' patterns of drug information use. [3] Physicians depended primarily on journals and texts for drug information [4] and board certification and hold-

\footnotetext{
"Conflict of Interest Declaration: The authors have no conflicts of interest to declare.

${ }^{\#}$ Corresponding author.
}

ing an academic appointment influenced their use [3].

Early studies have indicated that direct mail from pharmaceutical manufacturers was the most frequently used source of information by physicians [3], with neighboring countries such as Saudi Arabia [5] and the United Arab Emirates being no exceptions as these are a major source of information for physicians [6], since access to full text journal articles and access to resources is limited in these countries [1]. Reliance on these sources of information is problematic as these sources are commercially-oriented and make the information suspect [1]. Medical journals, continuing education programs, and physician colleagues were the most frequently consulted sources [3]. Physicians' use of drug information sources is strongly influenced by their attitudes toward use [4]. The Physician Desk Reference (PDR), medical textbooks, and newsletters have been found to be commonly used, with the PDR being viewed as the most accurate and accessible [3]. 
Although studies have evaluated the types of information used by physicians, they have provided conflicting results as the most readily used sources [3]. No study has been conducted on a group of physicians with different educational backgrounds as are practicing in the Emirate of Abu Dhabi in the United Arab Emirates.

Concern with higher cost of medical care [1,7], adverse drug reactions $[1,8]$, the influence of pharmaceutical companies on drug therapy [1,9], and the poor use of available expertise and useful medical information by people of the health profession $[1,10,11]$ is a concern of health governing bodies in the United Arab Emirates.

In the United Arab Emirates, healthcare is regulated by the Federal government body known as the Ministry of Health. Health Authority-Abu Dhabi is the local government branch which is responsible for regulating the healthcare industry and developing Abu Dhabi's health policy. The country operates under a government subsidized healthcare program for all legal residents.

This study objective was to determine physicians' preference for different drug information sources by surveying a diverse group of expatriate physicians licensed to practice in the Emirate of Abu Dhabi in the United Arab Emirates.

\section{Methods}

The study was conducted in the Emirate of Abu Dhabi in the UAE by the Health Authority Abu Dhabi Poison \& Drug Information Center (HAAD-PDIC). A proposal was submitted and approved by the HAAD Research \& Ethics Board as well as the HAAD Licensing Department which granted us access to the physician licensing files which contained all pertinent information including their email addresses.

A questionnaire was designed to collect information on physicians' perception of drug information resources. The survey questions consisted of three sections. The first section consisted of general demographic information. The second section focused on physicians' current resource utilization practice, and the third section assessed their preferences.

The questionnaire was submitted electronically to Health Authority-Abu Dhabi registered physicians with correct email addresses $(n=3224)$ through the period of April 1 to May 13, 2012 and requested to forward the completed survey to the investigators.

A total of 3463 licensed physicians were identified from a comprehensive list of healthcare professionals emails provided by the HAAD licensing department. An initial email was sent out followed by two reminder emails that were sent at 2-week intervals. A total of 239 emails were sent back to as rejections; hence, we assumed that 3224 physicians received the request for survey participation.
A total of 431 started the survey but only 372 physicians completed the entire survey with an overall response rate of $11.54 \%$. A prior analysis based hierarchical multiple regression showed that the sample size (372 out of 3224 physicians who received the survey) had a $95 \%$ confidence level and was within a 5\% margin of error.

Minitab software was used in the statistical analysis of results. Descriptive statistics such as frequency distributions were obtained. Pearson Chi square test was used to find the correlation between qualitative variables at the $5 \%$ significance level. A p-value of less than 0.05 represents a significant difference.

\section{Results}

A total of 431 physicians registered to participate in the electronic survey with 372 completing it entirely.

\subsection{Demographics}

Demographic information for the surveyed physicians is provided in Table 1. Respondents were employed at government hospitals $40.05 \%$ and private hospitals 59.95\%. Most of the respondents were from the Indian Sub-Continent (30.11\%), followed by Middle East (26.08\%), Africa (24.76\%), Europe/USA/Australia (17.74\%), and Asian (1.34\%). The respondents consisted of $31.45 \%$ female and $68.55 \%$ male; with varying experience, consultants

Table 1. Demographic information for surveyed physicians.

\begin{tabular}{|c|c|}
\hline Demographics & n (\%) \\
\hline \multicolumn{2}{|l|}{ Gender $(n=372)$} \\
\hline Male & $255(68.55)$ \\
\hline Female & 117 (31.45) \\
\hline \multicolumn{2}{|l|}{ Age distribution $(\mathrm{n}=372)$} \\
\hline 68 or older & $25(6.72)$ \\
\hline $58-67$ & $61(16.4)$ \\
\hline $48-57$ & $120(32.26)$ \\
\hline $38-47$ & 107 (28.76) \\
\hline Less than $37 \mathrm{yr}$ & 59 (15.86) \\
\hline \multicolumn{2}{|l|}{ Healthcare facility $(\mathrm{n}=372)$} \\
\hline Government & 149 (40.05) \\
\hline Private & $223(59.95)$ \\
\hline \multicolumn{2}{|l|}{ Nationality $(n=372)$} \\
\hline Europe/USA/Australia & 66 (17.74) \\
\hline Indian Sub-Continent & $112(30.11)$ \\
\hline Middle East & 97 (26.08) \\
\hline Africa & 92 (24.73) \\
\hline Asian & $5(1.34)$ \\
\hline \multicolumn{2}{|l|}{ Qualifications ( $\mathrm{n}=372$ ) } \\
\hline Consultant Physician & $83(22.31)$ \\
\hline Specialist Physician & $134(36.02)$ \\
\hline Medical Practitioner & 155 (41.67) \\
\hline
\end{tabular}


22.31\%, specialist physicians 36.02\%, and medical practitioners $41.67 \%$. Age distribution range was from less than 37 years to 68 years or older.

\subsection{Current Practice}

Preference of online versus hardcopy sources, respondents overwhelmingly choose online (74\%) and hardcopy (26\%). A correlation between age or qualifications and preference of online versus hardcopy sources did not show statistical significance $(\mathrm{P}-$ Value $=0.253$ and $\mathrm{P}$ Value $=0.500$, respectively). Government hospitals preferred online resources over private hospitals (118 (79.73\%) vs $156(70.27 \%)$ ) with a significant p-value of 0.042 . A preference of online resources compared to hardcopy between Consultant physician 69 (83.13\%), Specialist Physician 101 (75.94\%), and Medical Practitioner 104 (67.53\%) was statistically significant at a p-value of 0.027. Physicians from Europe/USA/Australia spent less time compared to other nationalities utilizing drug information resources with at a p-value of 0.013 .

Relationship between qualifications and number of references chosen (up to 3 resources versus 3 references or more) was statistically significant $(\mathrm{P}-\mathrm{Value}=0.000)$.

For broader sources of information, respondents overwhelmingly choose continuing medical education (CME) (46\%), followed by drug information (28\%), diagnosis/patient information (20\%, and peer-to-peer interactions (7\%).

When asked about most used drug information sources, respondents selected British National Formulary, followed by Package Inserts, UpToDate, Drugs in Pregancy \& Lactation, etc. (see complete listing in Figure 1 below).

\subsection{Resource Preferences}

When asked which drug information resources physicians would like to have access to, respondents choose BNF, followed by UpToDate, MD Consult, and Drugs in Pregnancy \& Lactation, Physicians Desk Reference, Micromedex, Drugs Facts \& Comparisons, etc. (see complete listing in Figure 2).

Thirty percent of the physicians surveyed stated that a particular source in the HAAD PDIC would encourge them to contact the center, while $10.5 \%$ would refrain, and $59.4 \%$ remained neutral. The center was contacted by $28 \%$ of respondents.

\section{Discussion}

This study is the first attempt at identifying the drug information sources most commonly utilized and sought after by the physician population in the Emirate of Abu Dhabi. The majority of the respondents were male (68.55\%) and females (31.45\%), which are expected as this reflects the global male and female proportionality that exists among physicians [12,13]. However, there were some surprising findings as well.

For instance, a previous study conducted in Asian countries ranked drug information first followed by CME as the primary source of preferred drug information by physicians [14]. However, in this study CME ranked $46 \%$ versus $28 \%$ for drug information [12], in light of the fact that drug information tends to be more detailed and focused [15]. There may be several reasons for this finding. One reason is that $\mathrm{CME}$ is a requirement of the Health Authority Abu Dhabi for licensed physicians, in addition to a requirement of expatriate physicians (which comprise $93 \%$ of licensed physicians) in their countries

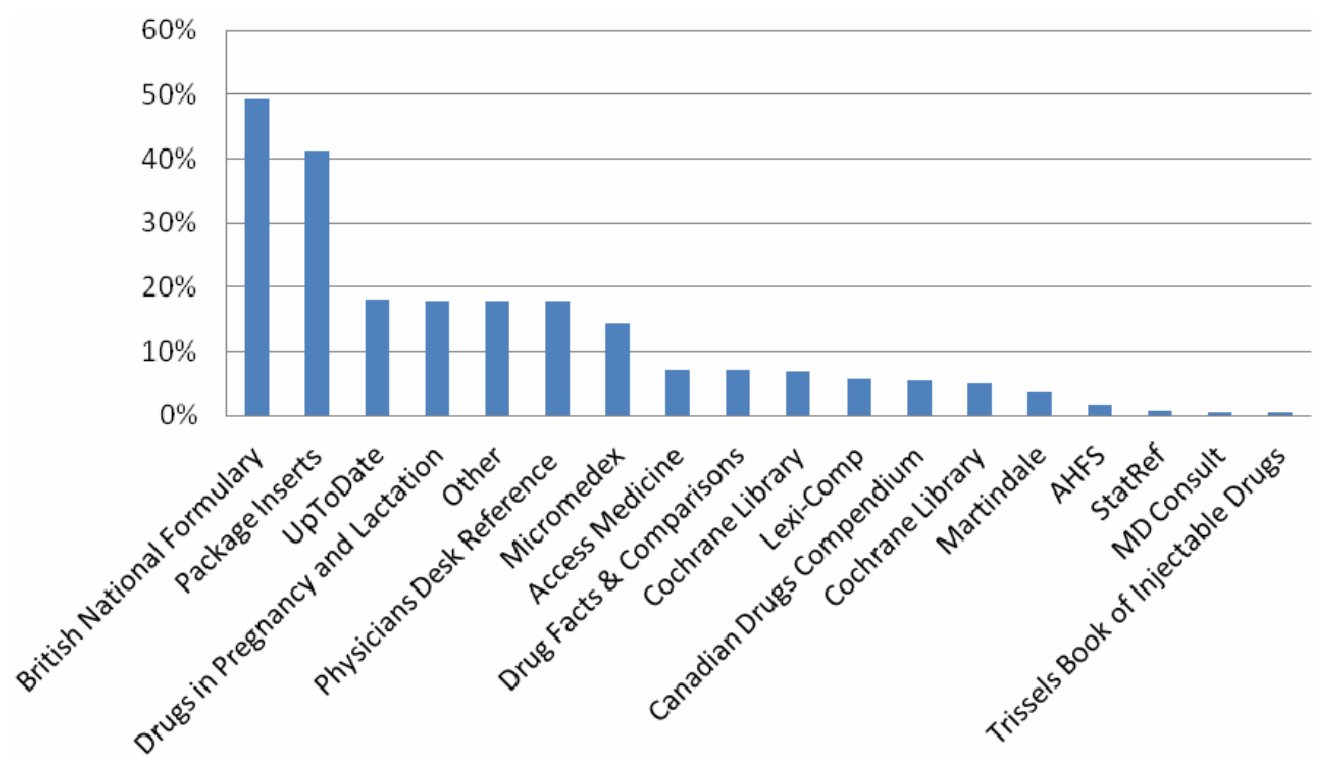

Figure 1. Percentage of most used drug information resources. 


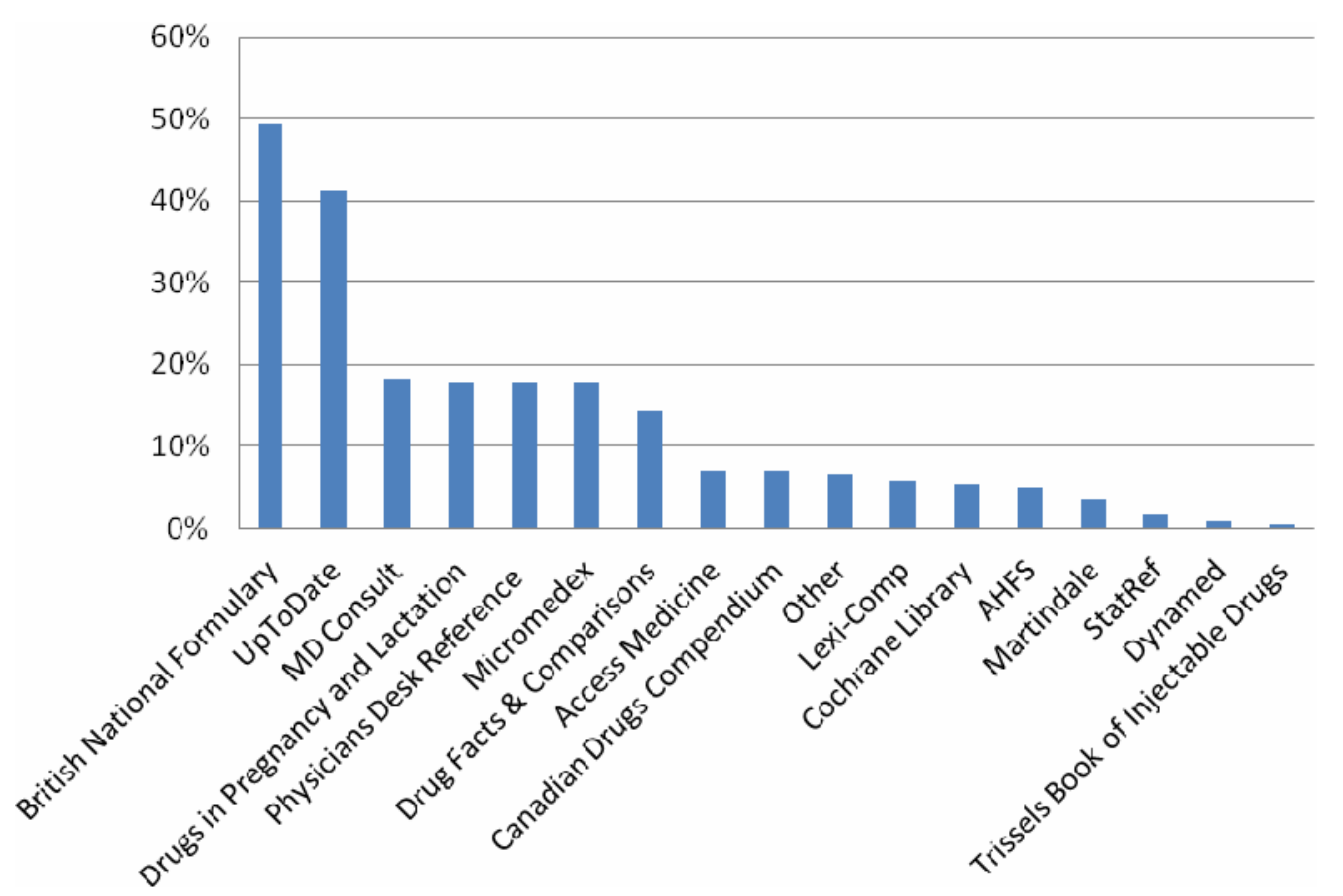

Figure 2. Percentage of drug resources physicians would like to access.

of origin to maintain their licensure. Access to CME is free of cost and convenient as it is widely available through various channels i.e. Health Authority Abu Dhabi CME Program, Pharmaceutical sponsored programs, and through academia. Lastly, although physician experiences with pharmacists was quite positive [16], in a study conducted in a nearby country, only $54 \%$ of physicians agreed that pharmacists were a reliable source of information [15].

Physician preferences for the British National Formulary, UpToDate, and package inserts as their favored sources of information, was not a surprising finding. UpToDate and the British National Formulary not only provide drug information but also treatment guidelines, which are necessary for physicians during their day-today practice. Package inserts as a top choice was selected probably due its availability with each drug product container, making it an easily accessible source.

Physicians from Europe/USA/Australia spent less time compared to other nationalities utilizing drug information resources with at a p-value of 0.013 . The reason for this finding may be due to their educational programs which may rely more on their knowledge and training compared to other training programs which rely more heavily on drug information resources [6]. In Western countries, primary care physicians use their colleagues to help them evaluate and validate the medical development about which they read $[4,6,17]$. They also rely on peers who they feel are better informed about medical advances; therefore, they seek their advice and opinion regarding a multitude of issues including diagnosis and drug treat- ment $[4,6,17]$.

Thirty percent of respondents stated that they would contact the PDIC if it stocked a particular source, but only $28 \%$ have previously contacted the center. This may indicate that one-third of the physicians surveyed may not be aware of the full extent of drug information sources available at the center.

It is also interesting to note that although internet access and use of computers is more common, only $74 \%$ of respondents prefer to use online sources of information. This may be attributed to a segment of older physicians (21\% of the respondents ages ranged from 58 - 77 years) who may still not be as comfortable with using computers as younger physicians.

The most commonly used resource by respondents was the British National Formulary and package inserts which appear to be more easily accessible and conveniently available on hand.

\section{Conclusion}

This survey showed that although the physician population can be described as a mix of different approaches to medicine, there are surprising few differences with regard to preferences and utilization of drug information resources. Continuing medical education requirements, availability of online versus hardcopy resources, access to some resources, and usage of the drug information center will continue to drive drug information utilization practices. As a health regulator, the Health AuthorityAbu Dhabi can ensure that physicians are aware of the 
full extent of resources available to them at the HAAD Poison \& Drug Information Center, as well as stock the most widely used and preferred resources.

\section{REFERENCES}

[1] S. A. Osiobe, "Use of Information Resources by Health Professionals: A Review of the Literature," Social Science \& Medicine, Vol. 21, No. 9, 1985, pp. 965-973.

[2] A. Woodsworth and V. R. Neufield, "A Survey of Physician Self-Education Patterns in Toronto Part I Use of Libraries," Canadian Library Journal, Vol. 29, 1972, pp. 38-44.

[3] C. Gaither, R. P. Bagoui, D. M. Kirking and F. J. Ascione, "Factors Related to Physicians' Attitudes and Beliefs toward Drug Information Sources,” Drug Information Journal, Vol. 28, 1994, pp. 817-827. doi:10.1177/009286159402800318

[4] G. H. Smith, D. L. Sorby and L. J. Sharp, "Physicians' Attitudes toward Drug Information Resources,” American Journal of Hospital Pharmacy, Vol. 32, 1975, pp. 19-25.

[5] H. Gharibyar and Y. Sharif, "Evaluation of Pharmaceutical Drug Information Brochures in the Emirate of Abu Dhabi (United Arab Emirates)," Journal of Pharmaceutical Health Services Research, Vol. 3, No. 1, 2012, pp. 57-62. doi:10.1111/j.1759-8893.2011.00071.x

[6] H. S. Abou-Auda, "Information-Seeking Behavior and Attitudes of Physicians toward Drug Information Resources and Services in Saudi Arabia: A Nationwide Survey,” Saudi Medical Journal, Vol. 29, No. 1, 2008, pp. 107-115.

[7] G. L. Oppenheim, S. H. Erickson and C. Ashworth, "The Family Physician's Knowledge of the Cost of Prescribed Drugs,” Journal of Family Practice, Vol. 12, No. 6, 1981, pp. 1027-1030.
[8] M. J. Kupst, et al., "Evaluation of Methods to Improve Communication in the Physician-Patient Relationship," American Journal of Orthopsychiatry, Vol. 45, No. 3, 1975, pp. 420-429.

[9] E. Hemminki, "Review of Literature on the Factors Affecting Drug Prescribing," Social Science \& Medicine, Vol. 9, No. 2, 1975, pp. 111-116.

[10] R. A. Floyd and R. Taketoma, "Serum Drug Level Utilization Review-A Clinical Pharmacy Service,” Drug Intelligence \& Clinical Pharmacy, Vol. 13, 1968, 99-105.

[11] S. Henley, W. Tester and R. Knapp, "Dissemination of Drug Information,” Hospitals, Vol. 42, No. 12, 1968, pp. 99-105.

[12] World Health Organization and Global Health Observatory (GHO), "Male to Female Ratio of Physicians Density per 1000 Population, Latest Available Year,” 2012. http://www.who.int/gho/health_workforce/physicians_de nsity_gender_text/en/index.html

[13] R. H. Wharrad, "The Global Distribution of Physicians and Nurses," Journal of Advanced Nursing, Vol. 30, No. 1, 1999, pp. 109-130.

[14] How Healthcare Professionals Perceive Medical Informaiton, "Medical Information \& Professional Networking HCP Survey,” UBM Medica Asia, 2012.

[15] J. P. Gettig, J. K. Jordan and A. H. Sheehan, “A Survey of Current Perceptions of Drug Information Practice and Training," Hospital Pharmacy, Vol. 44, No. 4, 2009, pp. 325-331. doi:10.1310/hpj4404-325

[16] W. E. Smith, M. D. Ray and D. M. Shannon, "Physician’s Expectations of Pharmacists," American Journal of Health-System Pharmacy, Vol. 59, No. 1, 2002, pp. 50-57.

[17] A. D. Weinberg, L. Ullian and W. D. Richards, "Informed Advice- and Information-Seeking between Physicians," Journal of Medical Education, Vol. 56, 1981, pp. 174180. 